\title{
Dynamic and geometric phase accumulation by Gaussian-type modes in first-order optical systems
}

\author{
Tatiana Alieva ${ }^{1, *}$ and Martin J. Bastiaans ${ }^{2}$ \\ ${ }^{1}$ Facultad de Ciencias Físicas, Universidad Complutense de Madrid, Ciudad Universitaria s/n, \\ Madrid 28040, Spain \\ ${ }^{2}$ Faculteit Elektrotechniek, Technische Universiteit Eindhoven, Postbus 513, 5600 MB Eindhoven, Netherlands \\ *Corresponding author: talieva@fis.ucm.es
}

Received April 15, 2008; revised June 6, 2008; accepted June 19, 2008; posted June 25, 2008 (Doc. ID 95028); published July 22, 2008

Based on the ray transformation matrix formalism, we propose a simple method for the identification of the dynamic and geometric parts of the Gouy phase, acquired by an appropriate Gaussian-type beam while propagating through a first-order optical system. (C) 2008 Optical Society of America

OCIS codes: 070.2575, 070.2580, 070.2590, 070.3185, 070.4690, 080.2468.

A transversal mode with a Gaussian envelope, undergoing a cycle of transformations while propagating through a paraxial optical system, accumulates Gouy phase, usually divided into two parts: A dynamic part and a geometric part [1-4]. The identification of the Gouy phase is important in resonator theory [5], in optical trapping [6], and in possible applications of its geometric part for quantum computation [7]. The physical origin of the geometric phase, its calculation in parametric space $[2,3]$, and its experimental measurement [4] have been discussed in a series of publications.

In this Letter we propose a simple method for the determination of the Gouy phase - and in particular its dynamic and geometric parts-accumulated by an appropriate Gaussian-type mode during its propagation through a first-order optical system. It is based on the analysis of the eigenvalues of the ray transformation matrix associated with the first-order optical system.

Strictly speaking, a coherent beam of light $\Psi(\mathbf{r})$, propagating through an optical system described by an operator $\mathcal{R}$, accumulates a phase shift only if it is an eigenfunction of $\mathcal{R}$ with a unimodular eigenvalue $\exp (i \varphi): \quad \mathcal{R}\left[\Psi\left(\mathbf{r}_{i}\right)\right]\left(\mathbf{r}_{o}\right)=\exp (i \varphi) \Psi\left(\mathbf{r}_{o}\right)$. Therefore we need to identify a system where phase accumulation is possible and determine the corresponding beams.

Beam propagation through a lossless first-order optical system is described by the canonical integral transformation $\mathcal{R}^{\mathbf{T}}[8,9]$, whose kernel is parameterized by the real symplectic ray transformation matrix $\mathbf{T}$, which relates the (properly normalized) position $\mathbf{r}=(x, y)^{t}$ and direction $\mathbf{p}=\left(p_{x}, p_{y}\right)^{t}$ of an incoming ray to those of the outgoing ray. Using the modified Iwasawa decomposition [10] the (normalized) ray transformation matrix $\mathbf{T}$ can be written as a product of three symplectic matrices,

$$
\mathbf{T}=\left[\begin{array}{cc}
\mathbf{I} & \mathbf{0} \\
-\mathbf{G} & \mathbf{I}
\end{array}\right]\left[\begin{array}{cc}
\mathbf{S} & \mathbf{0} \\
\mathbf{0} & \mathbf{S}^{-1}
\end{array}\right]\left[\begin{array}{cc}
\mathbf{X} & \mathbf{Y} \\
-\mathbf{Y} & \mathbf{X}
\end{array}\right]=\mathbf{T}_{L} \mathbf{T}_{S} \mathbf{T}_{O}
$$

where the first matrix $\mathbf{T}_{L}$ represents a lens described by the symmetric matrix $\mathbf{G}=-\left(\mathbf{C A}^{t}+\mathbf{D B} \mathbf{B}^{t}\right)\left(\mathbf{A A}^{t}\right.$ $\left.+\mathbf{B B}^{t}\right)^{-1}$, the second matrix $\mathbf{T}_{S}$ corresponds to a scaler described by the positive definite symmetric matrix $\mathbf{S}=\left(\mathbf{A} \mathbf{A}^{t}+\mathbf{B} \mathbf{B}^{t}\right)^{1 / 2}$, and the third matrix $\mathbf{T}_{O}$ is orthogonal and can be described by the unitary matrix $\mathbf{U}=\mathbf{X}+i \mathbf{Y}=\left(\mathbf{A A}^{t}+\mathbf{B B}^{t}\right)^{-1 / 2}(\mathbf{A}+i \mathbf{B})$.

A simple example of Gouy phase accumulation is the propagation of the well-known HermiteGaussian (HG) modes $\mathcal{H}_{m, n}(\mathbf{r})=\mathcal{H}_{m}(x) \mathcal{H}_{n}(y)$, where $\mathcal{H}_{n}(x)=2^{1 / 4}\left(2^{n} n !\right)^{-1 / 2} H_{n}(\sqrt{2 \pi x}) \exp \left(-\pi x^{2}\right)$ and $H_{n}(\cdot)$ denote the Hermite polynomials, through the fractional Fourier transformer (FT) described by the orthogonal $4 \times 4$ ray transformation matrix $\mathbf{T}_{f}\left(\gamma_{x}, \gamma_{y}\right)$ or, equivalently, by the unitary $2 \times 2$ matrix $\mathbf{U}_{f}\left(\gamma_{x}, \gamma_{y}\right)$,

$$
\mathbf{U}_{f}\left(\gamma_{x}, \gamma_{y}\right)=\left[\begin{array}{cc}
\exp \left(i \gamma_{x}\right) & 0 \\
0 & \exp \left(i \gamma_{y}\right)
\end{array}\right] \text {. }
$$

The separable fractional FT with fractional angles $\gamma_{x}$ and $\gamma_{y}$ of a function $f\left(\mathbf{r}_{i}\right)$ is defined as

$$
\mathcal{R}^{\mathbf{T}_{f}\left(\gamma_{x}, \gamma_{y}\right)}\left[f\left(\mathbf{r}_{i}\right)\right]\left(\mathbf{r}_{o}\right)=\iint K_{f}^{\gamma_{x}, \gamma_{y}}\left(\mathbf{r}_{i}, \mathbf{r}_{o}\right) f\left(\mathbf{r}_{i}\right) \mathrm{d} \mathbf{r}_{i},
$$

with $K_{f}^{\gamma_{x}}, \gamma_{y}\left(\mathbf{r}_{i}, \mathbf{r}_{o}\right)=K_{f}^{\gamma_{x}}\left(x_{i}, x_{o}\right) K_{f}^{\gamma_{y}}\left(y_{i}, y_{o}\right)$, where in optics the kernel $K_{f}^{\alpha}$ is usually chosen such that $[11,12]$

$$
\begin{aligned}
& K_{f}^{\alpha}\left(x_{i}, x_{o}\right) \exp (i \alpha / 2) \\
& \quad=\frac{\exp (i \alpha / 2)}{(i \sin \alpha)^{1 / 2}} \exp \left[i \pi \frac{\left(x_{i}^{2}+x_{o}^{2}\right) \cos \alpha-2 x_{i} x_{o}}{\sin \alpha}\right],
\end{aligned}
$$

$K_{f}^{0}\left(x_{i}, x_{o}\right)=\delta\left(x_{i}-x_{o}\right)$, and $K_{f}^{ \pm \pi}\left(x_{i}, x_{o}\right)=\mp i \delta\left(x_{i}+x_{o}\right)$. It is easy to see that for $\alpha= \pm \pi / 2$ the kernel $K_{f}^{\alpha}\left(x_{i}, x_{o}\right)$ reduces to the common direct/inverse FT apart from the constant factor $( \pm i)^{-1 / 2}$. Note the $4 \pi$ periodicity in the fractional angle $\alpha$.

The HG modes $\mathcal{H}_{m, n}$ are eigenfunctions of the separable fractional FT; for the kernel that describes the propagation of light [see Eqs. (3) and (4)] the eigenvalues read $\exp \left\{-i\left[\left(m+\frac{1}{2}\right) \gamma_{x}+\left(n+\frac{1}{2}\right) \gamma_{y}\right]\right\}$. We note that the acquired Gouy phase $-\left[\left(m+\frac{1}{2}\right) \gamma_{x}+\left(n+\frac{1}{2}\right) \gamma_{y}\right]$ is defined by the unimodular eigenvalues $\exp \left(i \gamma_{x}\right)$ and $\exp \left(i \gamma_{y}\right)$ of the unitary matrix $\mathbf{U}_{f}\left(\gamma_{x}, \gamma_{y}\right)$ [see Eq. (2)]. 
A separable fractional FT can be written as the cascade of a symmetric and an antisymmetric one, associated with the unitary matrices $\mathbf{U}_{f}(\mu, \mu)=\exp (i \mu) \mathbf{I}$ and $\mathbf{U}_{f}(\gamma,-\gamma)$, respectively,

$$
\mathbf{U}_{f}\left(\gamma_{x}, \gamma_{y}\right)=\mathbf{U}_{f}(\mu, \mu) \mathbf{U}_{f}(\gamma,-\gamma)=\exp (i \mu) \mathbf{U}_{f}(\gamma,-\gamma),
$$

where $\gamma_{x}=\mu+\gamma$ and $\gamma_{y}=\mu-\gamma$. Note that $\operatorname{det} \mathbf{U}_{f}(\gamma,-\gamma)=1$, whereas $\operatorname{det} \mathbf{U}_{f}(\mu, \mu)=\exp (2 i \mu)$ is only unity for $\mu=k \pi$. The special case of $\mu$ being an odd integer multiple of $\pi$ will be discussed later.

The decomposition (5) is crucial for the identification of the dynamic and geometric parts of the Gouy phase. Indeed, during its propagation through a symmetric fractional FT, the $\mathrm{HG}$ mode $\mathcal{H}_{m, n}$ acquires the dynamic phase $-(m+n+1) \mu$, defined by the sum of mode indices, whereas in the case of the antisymmetric fractional FT, the accumulated phase-known as the geometric phase-is proportional to the index difference $-(m-n) \gamma$.

Based on the dynamic and geometric phase accumulation by $\mathrm{HG}$ modes, other sets of orthonormal modes can be found together with the corresponding optical systems. It has been shown in [13] that any real symplectic ray transformation matrix $\mathbf{T}$ with unimodular complex eigenvalues is similar to the matrix $\mathbf{T}_{f}$ of a separable fractional $\mathrm{FT}$ in the sense that $\mathbf{T}=\mathbf{M} \mathbf{T}_{f}\left(\gamma_{x}, \gamma_{y}\right) \mathbf{M}^{-1}$, where $\mathbf{M}$ is a real symplectic ray transformation matrix and can thus be associated with a certain first-order optical system. The parameters $\gamma_{x}$ and $\gamma_{y}$ are obtained from the unimodular eigenvalues $\exp \left( \pm i \gamma_{x}\right)$ and $\exp \left( \pm i \gamma_{y}\right)$ of the matrix $\mathbf{T}$, and the matrix $\mathbf{M}$ is constructed from the eigenvectors of $\mathbf{T}$.

If the HG mode $\mathcal{H}_{m, n}$ is applied at the input of a system with ray transformation matrix $\mathbf{M}$, we obtain the Gaussian-type mode $\mathcal{H}_{m, n}^{\mathbf{M}}=\mathcal{R}^{\mathbf{M}_{\mathcal{H}}} \mathcal{H}_{m, n}$ at its output. An explicit form for this mode can be found in $[14,15]$. The modes $\mathcal{H}_{m, n}^{\mathbf{M}}$ for different $m$ and $n$ (but with the same $\mathbf{M}$ ) form a complete orthonormal set. While the mode $\mathcal{H}_{m, n}^{\mathbf{M}}$ propagates through the system described by the matrix $\mathbf{M} \mathbf{T}_{f}\left(\gamma_{x}, \gamma_{y}\right) \mathbf{M}^{-1}$, for which system it is an eigenfunction [14], it accumulates the Gouy phase $-\left[\left(m+\frac{1}{2}\right) \gamma_{x}+\left(n+\frac{1}{2}\right) \gamma_{y}\right]=-(m+n+1) \mu-(m-n) \gamma$, in which we recognize the dynamic phase and the geometric phase, corresponding to the symmetric part $\mathbf{U}_{f}(\mu, \mu)$ and the antisymmetric part $\mathbf{U}_{f}(\gamma,-\gamma)$ of the separable fractional FT, respectively; cf. Eq. (5). Thus we can conclude that if a ray transformation matrix $\mathbf{T}$ has unimodular complex eigenvalues then its eigenvectors define first $\mathbf{M}$ and subsequently the Gaussian-type modes $\mathcal{H}_{m, n}^{\mathbf{M}}$, which accumulate the Gouy phase directly related to the eigenvalues of $\mathbf{T}$. Linear combinations of the $\mathcal{H}_{m, n}^{\mathbf{M}}$ modes with the same sum $m+n$ or difference $m-n$ of the indices acquire the same dynamic or geometric phase, respectively, while propagating through the appropriate system.

Using again the modified Iwasawa decomposition for the ray transformation matrix $\mathbf{M}=\mathbf{M}_{L} \mathbf{M}_{S} \mathbf{M}_{O}$ [see Eq. (1)], we observe that the mode $\mathcal{H}_{m, n}^{\mathbf{M}}$ differs from
$\mathcal{H}_{m, n}^{\mathbf{M}_{O}}$ only by an appropriate scaling and quadraticphase modulation. We can thus limit our consideration to the modes that are generated by canonical transformations that correspond to orthogonal ray transformation matrices. Note that any orthogonal real symplectic matrix is similar to the ray transformation matrix of a separable fractional FT and therefore is associated with the unitary matrix $\mathbf{U}=\mathbf{U}_{0} \mathbf{U}_{f}\left(\gamma_{x}, \gamma_{y}\right) \mathbf{U}_{0}^{-1}$.

In addition to the symmetric and antisymmetric fractional FTs there are two other basic transformations described by orthogonal ray transformation matrices: The rotator and the gyrator, associated with the unitary matrices

$$
\mathbf{U}_{r}(\gamma)=\left[\begin{array}{cc}
\cos \gamma & \sin \gamma \\
-\sin \gamma & \cos \gamma
\end{array}\right], \quad \mathbf{U}_{g}(\gamma)=\left[\begin{array}{cc}
\cos \gamma & i \sin \gamma \\
i \sin \gamma & \cos \gamma
\end{array}\right],
$$

respectively. The rotator satisfies the additivity property $\mathbf{U}_{r}\left(\gamma_{1}\right) \mathbf{U}_{r}\left(\gamma_{2}\right)=\mathbf{U}_{r}\left(\gamma_{1}+\gamma_{2}\right)$ and hence $\mathbf{U}_{r}^{-1}(\gamma)$ $=\mathbf{U}_{r}(-\gamma)$, and similar relations hold for the gyrator $\mathbf{U}_{g}(\gamma)$ and for the symmetric and antisymmetric fractional FTs $\mathbf{U}_{f}(\gamma, \gamma)$ and $\mathbf{U}_{f}(\gamma,-\gamma)$. We remark that all these systems have a rotating character: The rotator performs a rotation in the $(x, y)$ and $\left(p_{x}, p_{y}\right)$ planes, the separable fractional FT in the $\left(x, p_{x}\right)$ and $\left(y, p_{y}\right)$ planes (possibly with different angles), and the gyrator in the mixed $\left(x, p_{y}\right)$ and $\left(y, p_{x}\right)$ planes. Moreover $\operatorname{det} \mathbf{U}_{r}(\gamma)=\operatorname{det} \mathbf{U}_{g}(\gamma)=\operatorname{det} \mathbf{U}_{f}(\gamma,-\gamma)=1$, and the rotator and the gyrator are similar to the antisymmetric fractional FT,

$$
\begin{aligned}
& \mathbf{U}_{r}(\gamma)=\mathbf{U}_{g}(-\pi / 4) \mathbf{U}_{f}(-\gamma, \gamma) \mathbf{U}_{g}(\pi / 4), \\
& \mathbf{U}_{g}(\gamma)=\mathbf{U}_{r}(-\pi / 4) \mathbf{U}_{f}(\gamma,-\gamma) \mathbf{U}_{r}(\pi / 4)
\end{aligned}
$$

The helicoidal Laguerre Gaussian (LG) mode $\mathcal{H}_{m, n}^{\mathbf{M}_{g}(-\pi / 4)}$ may thus be obtained from the HG mode by a gyrator transformation with the angle $-\pi / 4$. It is an eigenfunction of the rotator $\mathbf{U}_{r}(\gamma)$ and therefore accumulates the geometric phase $-(m-n) \gamma$ while propagating through the rotator system. Analogously, the HG mode rotated at $-\pi / 4, \mathcal{H}_{m, n}^{\mathbf{M}_{r}(-\pi / 4)}$, is an eigenfunction of the gyrator $\mathbf{U}_{g}(\gamma)$ with eigenvalue $\exp [-i(m-n) \gamma]$.

From the commutation relation $\mathbf{U U}_{f}(\mu, \mu)$ $=\mathbf{U}_{f}(\mu, \mu) \mathbf{U}$ we easily conclude that any mode obtained from the $\mathrm{HG}$ mode by a canonical integral transformation associated with an orthogonal ray transformation matrix accumulates dynamic phase while propagating through a symmetric fractional FT.

Several optical systems to realize fractional FTs have been proposed $[11,16]$. Based on the matrix formalism, flexible optical setups have been designed [17], with which an antisymmetric fractional FT $\mathcal{R}^{\mathbf{T}_{f}(\gamma,-\gamma)}$, a gyrator $\mathcal{R}^{\mathbf{T}_{g}(\gamma)}$, and a rotator $\mathcal{R}^{\mathbf{T}_{r}(\gamma)}$ (with $\pi / 2 \leqslant \gamma \leqslant 3 \pi / 2$ ) can be realized, and which can be used for controlled geometric phase accumulation.

Let us now consider the transformation described by the matrix $\mathbf{M}=-\mathbf{I}$, which physically produces a 
beam rotation through an angle $\pi$ in the $x y$ plane. This case is interesting for two reasons. First, because not only the generalized Gaussian modes, but all beams for which $f(\mathbf{r})=f(-\mathbf{r})$ or $f(\mathbf{r})=-f(-\mathbf{r})$, are self-reciprocal for this transformation; and second, because the $\pi$ rotation can be performed by two principally different kinds of systems: One is similar to the symmetric fractional FT $\mathbf{T}_{f}(\pi, \pi)$ and another one is similar to the antisymmetric fractional FT $\mathbf{T}_{f}(\pi,-\pi)$. Although in matrix form these two transformations look the same, they are different in the integral representation due to the additional factor -1 in the case of the symmetric fractional FT. The propagation through a rotationally symmetric system, for example a $4-f$ arrangement with which we realize a cascade of two $(\pi / 2, \pi / 2)$ FTs, leads to dynamic phase accumulation, whereas the propagation through a system with astigmatic lenses, with which we realize a cascade of two antisymmetric $(\pi / 2,-\pi / 2)$ FTs, leads to geometric phase acquirement, and the results have different signs.

If the eigenvalues of the matrix are not unimodular we can speak about phase accumulation only in a wide sense, where scaling and quadratic-phase modulation are present. In this case we permit the beam to be an eigenfunction of the transformation described by the orthosymplectic matrix in the Iwasawa decomposition (1). Thus, after having performed the Iwasawa decomposition (1) for the system's ray transformation matrix we determine the eigenvalues of the unitary matrix $\mathbf{U}$ that describes the orthosymplectic part, and represent $\mathbf{U}$ in the form $\mathbf{U}=\exp (i \mu) \mathbf{U}_{0} \mathbf{U}_{f}(\gamma,-\gamma) \mathbf{U}_{0}^{-1}$. We can then conclude that the Gaussian mode $\mathcal{H}_{m, n}^{\mathbf{U}_{0}}(\mathbf{r})$, obtained from the HG mode $\mathcal{H}_{m, n}(\mathbf{r})$ by the canonical integral transformation parameterized by the matrix $\mathbf{U}_{0}$, accumulates a dynamic phase $-(m+n+1) \mu$ and a geometric phase $-(m-n) \gamma$ when it propagates through the system (in a wide sense, since we may have an additional scaling and/or quadratic-phase modulation).

As an example, we mention the propagation of $\mathrm{HG}$ and LG beams in free space. These beams are actually the eigenfunctions of the symmetric fractional Fourier transformation and not of the Fresnel transformation that describes free-space propagation in the paraxial approximation. However, during freespace propagation these beams preserve the form of their intensity distribution and differ from the original input beams only by some scaling (i.e., change of the beam width) and quadratic-phase modulation (i.e., change of the wavefront's radius of curvature). The corresponding ray transformation matrix is defined by $\mathbf{A}=\mathbf{D}=\mathbf{I}, \mathbf{B}=s \mathbf{I}$, and $\mathbf{C}=\mathbf{0}$, and thus $\mathbf{G}=s\left(1+s^{2}\right)^{-1 / 2} \mathbf{I}, \quad \mathbf{S}=\left(1+s^{2}\right)^{1 / 2} \mathbf{I}, \quad \mathbf{U}=\exp (i \arctan s) \mathbf{I}$, where $s=\lambda z / w^{2}$ with $\lambda$ being the wavelength, $z$ being the propagation distance, and $w$ being the beam waist. Then the mode $\mathcal{H}_{m, n}^{\mathbf{U}_{\circ}}$ accumulates only dy- namic phase, equal to $-(m+n+1) \arctan s$. The connection between the accumulation of dynamic phase during propagation of Gaussian modes through an isotropic first-order optical system and the action of a symmetric fractional FT was studied in [18].

We can conclude that rotations in phase space are associated with Gouy phase accumulation. Dynamic phase is accumulated in symmetric, rotationally invariant systems, which are similar to the symmetric fractional FT. Even the fundamental Gaussian mode accumulates dynamic phase during its propagation through such systems. Geometric phase accumulation requires a system with astigmatic elements, similar to the antisymmetric fractional FT, as well as mode asymmetry $(m \neq n)$. The analysis of the eigenvalues of the ray transformation matrix of a firstorder optical system permits us to identify these two phase contributions and to determine the selfreciprocal beams for the corresponding transformation.

T. Alieva acknowledges the Spanish Ministry of Education and Science (project TEC 2005-02180 /MIC). M. J. Bastiaans appreciates the hospitality at Universidad Complutense de Madrid.

\section{References}

1. S. J. van Enk, Opt. Commun. 102, 59 (1993).

2. M. J. Padgett and J. Courtial, Opt. Lett. 24, 430 (1999).

3. G. F. Calvo, Opt. Lett. 30, 1207 (2005).

4. E. J. Galvez, P. R. Crawford, H. I. Sztul, M. J. Pysher, P. J. Haglin, and R. E. Williams, Phys. Rev. Lett. 90, 203901 (2003).

5. A. E. Siegman, Lasers (University Science Books, 1986).

6. F. Gittes and C. F. Schmidt, Opt. Lett. 23, 7 (1998).

7. A. Ekert, M. Ericsson, P. Hayden, H. Inamori, J. A. Jones, D. K. L. Oi, and V. Vedral, J. Mod. Opt. 47, 2501 (2000).

8. S. A. Collins, Jr., J. Opt. Soc. Am. 60, 1168 (1970).

9. T. Alieva and M. J. Bastiaans, Opt. Lett. 30, 3302 (2005).

10. R. Simon and K. B. Wolf, J. Opt. Soc. Am. A 17, 342 (2000).

11. H. M. Ozaktas, Z. Zalevsky, and M. A. Kutay, The Fractional Fourier Transform with Applications in Optics and Signal Processing (Wiley, 2001).

12. A. C. McBride and F. H. Kerr, IMA J. Appl. Math. 39, 159 (1987)

13. M. J. Bastiaans and T. Alieva, J. Opt. Soc. Am. A 23, 1875 (2006)

14. T. Alieva and M. J. Bastiaans, Opt. Lett. 30, 1461 (2005).

15. T. Alieva and M. J. Bastiaans, Opt. Lett. 32, 1226 (2007).

16. A. Sahin, H. M. Ozaktas, and D. Mendlovic, Appl. Opt. 37, 2130 (1998).

17. J. A. Rodrigo, T. Alieva, and M. L. Calvo, J. Opt. Soc. Am. A 23, 2494 (2006).

18. R. Borghi, M. Santarsiero, and R. Simon, J. Opt. Soc. Am. A 21, 572 (2004). 